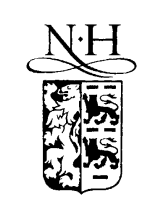

\title{
A stabilized formulation for incompressible elasticity using linear displacement and pressure interpolations
}

\author{
M. Chiumenti ${ }^{\text {a,* }}$, Q. Valverde ${ }^{\text {b }}$, C. Agelet de Saracibar ${ }^{\text {a }}$, M. Cervera ${ }^{\text {a }}$ \\ a Departamento de Resistència de Materials i Estructures a l'Enginyeria, Universitat Politècnica de Catalunya, Barcelona 08034, Spain \\ b Departamento de Ingeniería, Sección Igeniería Mecánica, Pontificia Universidad Católica del Perú, Lima, Peru
}

Received 7 February 2002; received in revised form 18 June 2002

\begin{abstract}
In this paper a stabilized finite element method to deal with incompressibility in solid mechanics is presented. A mixed formulation involving pressure and displacement fields is used and a continuous linear interpolation is considered for both fields. To overcome the Babuška-Brezzi condition, a stabilization technique based on the orthogonal sub-scale method is introduced. The main advantage of the method is the possibility of using linear triangular or tetrahedral finite elements, which are easy to generate for real industrial applications. Results are compared with standard Galerkin and Q1P0 mixed formulations for nearly incompressible problems in the context of linear elasticity.

(c) 2002 Elsevier Science B.V. All rights reserved.
\end{abstract}

Keywords: Incompressible elasticity; Sub-grid scales method; Stabilized finite element methods

\section{Introduction}

This paper proposes a possible solution to the problem of incompressibility or near incompressibility in solid mechanics in the framework of linear elasticity. The objective is to avoid the so called volumetric locking, an undesirable effect exhibited by all low order elements based on the standard Galerkin formulation. Many successful strategies to avoid volumetric locking based on both mixed and enhanced formulations can be found in the literature [7,9,10], but they generally fail in the case of linear triangular or tetrahedral elements due to the lack of satisfaction of the Babuška-Brezzi condition [1]. Other formulations have been proposed by Zienkiewicz et al. [12], Taylor [11], Oñate et al. [8], Klaas et al. [6] etc. to deal with such elements, mainly motivated by the fact that nowadays for real life geometries tetrahedral meshes are relatively easy to generate. The main effort in this work is the extension to solid mechanics problems of the stabilization technique proposed by Hughes in [5], the sub-grid scale approach. An equal order interpolation of the mixed pressure and displacements fields will be introduced followed by a decomposition of the unknowns into resolvable and sub-grid scales orthogonal to the finite element space, following the works of

\footnotetext{
${ }^{*}$ Corresponding author.

E-mail address: chiument@cimne.upc.es (M. Chiumenti).
} 
Codina [2,4] for the solution of the numerical instabilities due to the incompressibility constraint of the Stokes problem. The basic idea is to approximate the effect of the component of the continuous solution which cannot be captured by the finite element solution and is the cause of the volumetric locking.

In the next section the equations that define the mechanical problem including the condition of incompressibility will be presented, introducing also the mixed formulation based on displacement and pressure fields. In Section 3 the stabilization model using the sub-grid scale approach will be presented. Some numerical examples are shown in Section 4, comparing the results obtained with the present formulation with the standard Galerkin formulation and the well known mixed Q1P0 approach.

\section{Incompressibility problem in solid mechanics}

The linear elastic problem will be taken as the reference model to show the incompressibility phenomenon in solid mechanics. Let us begin introducing some standard notation, $\Omega$ stands for an open and bounded domain of $R^{n_{\mathrm{dim}}}$ and $\bar{\Omega}$ its closure, $n_{\mathrm{dim}}$ is the number of space dimensions, its boundary $\Gamma$ is considered split into two disjoint sets such that $\Gamma=\overline{\partial \Omega_{u} \cup \partial \Omega_{t}}$ and $\partial \Omega_{u} \cap \partial \Omega_{t}=\emptyset$. The space of square integrable functions in $\Omega$ is $L^{2}(\Omega)$, and $H^{m}(\Omega)$ is the space of functions of which its derivatives up to order $m \geqslant 0$ (integer) belong to $L^{2}(\Omega)$. The space $H_{0}^{m}(\Omega)$ consists of those functions that belong to $H^{m}(\Omega)$ and vanish on $\partial \Omega$. Bold characters are used to denote vector counterparts of the spaces and the inner product in $L^{2}(\Omega)$ is denoted by $(\cdot, \cdot)$. Hereafter, orthogonality will be understood with respect to this product.
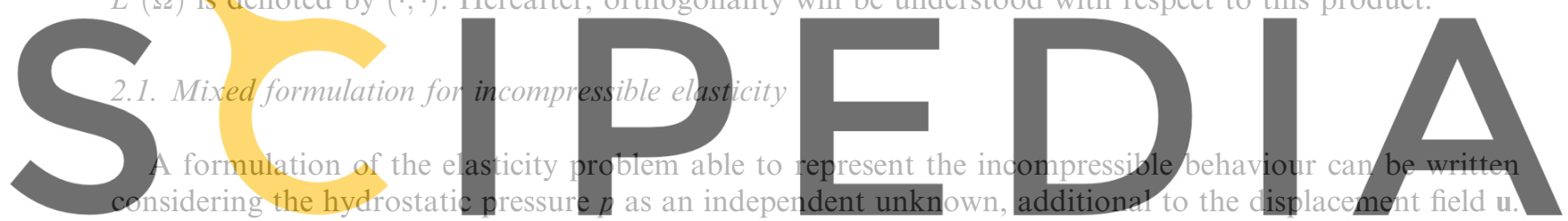

The stress tensor $\sigma$ can be expressed in terms of these two variables such as:

Register for frees at htteps/:ynww.scipedia.com to download the version without the watermark

$$
p=K \varepsilon_{\mathrm{v}}, \quad \text { with } \varepsilon_{\mathrm{v}}=\nabla \cdot \mathbb{u},
$$

where $\varepsilon_{\mathrm{v}}$ and $\operatorname{dev}\left[\nabla^{s} \mathbf{u}\right]$ are the volumetric and the deviatoric part of the deformation, respectively, $\mu$ is the Lamé constant referred to as the shear modulus and $K$ is the bulk modulus, also referred to as modulus of volumetric compressibility. As it can be observed, this constitutive equation is based on the decoupling of the deformation in its volumetric and deviatoric parts and it leads to a decoupled expression of the stress tensor as well. Making use of Eq. (1), momentum equation, together with Eq. (2) and the boundary conditions the problem can be formulated as: find the displacement field $\mathbf{u}: \bar{\Omega} \rightarrow R^{n_{\mathrm{dim}}}$ and the pressure field $p$, for prescribed body force per unit volume: $\mathbf{f}: \Omega \rightarrow R^{n_{\mathrm{dim}}}$ and $\overline{\mathbf{t}}: \partial \Omega_{t} \rightarrow R^{n_{\mathrm{dim}}}$, such that:

$$
\begin{aligned}
& \nabla p+2 \mu \nabla \cdot \operatorname{dev}\left[\nabla^{s} \mathbf{u}\right]+\mathbf{f}=\mathbf{0} \quad \text { in } \Omega, \\
& \frac{1}{K} p-\nabla \cdot \mathbf{u}=0 \quad \text { in } \Omega, \\
& \mathbf{u}=\mathbf{0} \quad \text { on } \partial \Omega_{u}, \\
& \boldsymbol{\sigma} \cdot \mathbf{n}=\overline{\mathbf{t}} \quad \text { on } \partial \Omega_{t} .
\end{aligned}
$$

Observe that the formulation is valid in both compressible and incompressible cases, within this context the incompressibility constraint is given by Eq. (4), which close to the limit $(K \rightarrow \infty)$ transforms into: 
$\nabla \cdot \mathbf{u}=0 \quad$ in $\Omega$

or, alternatively, $\varepsilon_{\mathrm{v}}=0$, with no volumetric change.

The variational statement of this problem consists in finding $(\mathbf{u}, p) \in \mathscr{V}_{0} \times \mathscr{Q}$, such that:

$$
\begin{array}{ll}
a(\mathbf{u}, \mathbf{v})+(p, \nabla \cdot \mathbf{v})=L(\mathbf{v}) & \forall \mathbf{v} \in \mathscr{V}_{0} \\
(\nabla \cdot \mathbf{u}, q)-\left(\frac{1}{K} p, q\right)=0 & \forall q \in \mathscr{Q},
\end{array}
$$

where the bilinear form $a(\mathbf{u}, \mathbf{v})$ and the operator $L(\mathbf{v})$ are given by

$$
\begin{aligned}
& a(\mathbf{u}, \mathbf{v})=\int_{\Omega} 2 \mu \operatorname{dev}\left[\nabla^{s} \mathbf{u}\right]: \nabla^{s} \mathbf{v} \mathrm{d} \Omega, \\
& L(\mathbf{v})=\int_{\Omega} \mathbf{f} \cdot \mathbf{v} \mathrm{d} \Omega+\int_{\partial \Omega_{t}} \mathbf{v} \cdot \overline{\mathfrak{t}} \mathrm{d} \Gamma .
\end{aligned}
$$

Observe that for the continuous problem the spaces for the displacement and pressure fields are $\mathscr{V}_{0}=\mathbf{H}_{0}^{1}(\Omega)$ and $\mathscr{Q}=L^{2}(\Omega)$, respectively. Finally, the discrete form of the problem is: find $\left(\mathbf{u}_{\mathrm{h}}, p_{\mathrm{h}}\right) \in$ $\mathscr{V}_{\mathrm{h}, 0} \times \mathscr{2}_{\mathrm{h}}$, such that:
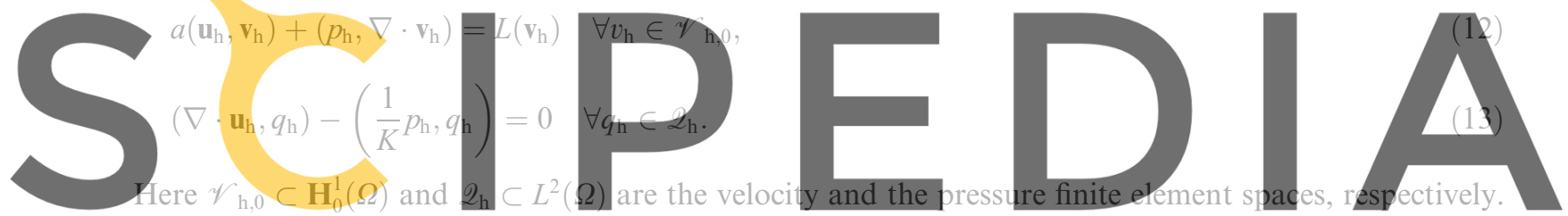

Observe that this means that, in principle, pressure fields may be discontinuous while displacement fields

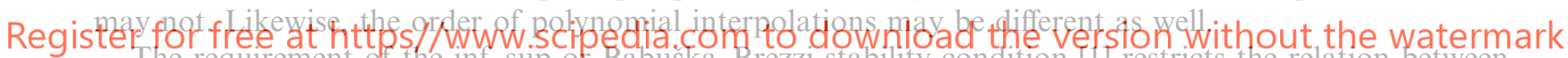
The requirement of the inf-sup of Babuska-Brezzi stability condition [1] restricts the relation between the interpolations of the involved fields, leading to the need of using different interpolations for u and $p$. Equal order $\mathbf{u} / p$ interpolations, such as convenient linear/linear interpolation, violate this condition and exhibit poor numerical performance and suffer high oscillations.

The objective of stabilized finite element formulations is to modify conveniently the problem expressed by (8) and (9) in order to define a method to overcome the requirement of the Babuška-Brezzi condition and, in particular, to make possible the use of equal order continuous interpolations.

In the following section we will introduce the sub-grid scale stabilization method. Hereafter, both displacements and pressure spaces are assumed to be built up using continuous finite element interpolations of the same order. It is worthwhile to define before some useful notation to rewrite the previous equations in a compact format.

Let $\mathbf{U}:=[\mathbf{u}, p]^{t}$ be the vector of $n_{\text {unk }}$ unknowns and $\mathscr{W}:=\left(H^{1}(\Omega)\right)^{n_{\mathrm{unk}}}$. The problem expressed by (3) and (4), together with the boundary conditions (5) and (6), can be stated as: find $\mathbf{U}:=[\mathbf{u}, p]^{t} \in \mathscr{W}_{0}=$ $\left(H_{0}^{1}(\Omega)\right)^{n_{\mathrm{unk}}}$, such that:

$$
\mathscr{L}(\mathbf{U}):=\left[\begin{array}{c}
-\nabla p-2 \mu \nabla \cdot \operatorname{dev}\left[\nabla^{s} \mathbf{u}\right] \\
-\frac{1}{K} p+\nabla \cdot \mathbf{u}
\end{array}\right]=\left[\begin{array}{l}
\mathbf{f} \\
0
\end{array}\right]=: \mathbf{F} .
$$

Furthermore, if $\mathbf{V}:=[\mathbf{v}, q]^{t} \in \mathscr{W}_{0}$, the variational statement for problem (14) can be written as:

$$
B(\mathbf{U}, \mathbf{V})=L(\mathbf{V}) \quad \forall \mathbf{V} \in \mathscr{W}_{0},
$$


where the bilinear form $B(\mathbf{U}, \mathbf{V})$, defined on $\mathscr{W}_{0} \times \mathscr{W}_{0}$, is:

$$
B(\mathbf{U}, \mathbf{V}):=a(\mathbf{u}, \mathbf{v})+(p, \nabla \cdot \mathbf{v})+(\nabla \cdot \mathbf{u}, q)-\left(\frac{1}{K} p, q\right)
$$

and operator $L(\mathbf{V})$ is given by:

$$
L(\mathbf{V})=\int_{\Omega} \mathbf{f} \cdot \mathbf{v} \mathrm{d} \Omega+\int_{\partial \Omega_{t}} \mathbf{v} \cdot \overline{\mathbf{t}} \mathrm{d} \Gamma .
$$

Likewise, the discrete counterpart of (15) is:

$$
B\left(\mathbf{U}_{\mathrm{h}}, \mathbf{V}_{\mathrm{h}}\right)=L\left(\mathbf{V}_{\mathrm{h}}\right) \quad \forall \mathbf{V}_{\mathrm{h}} \in \mathscr{W}_{\mathrm{h}, 0}
$$

being $\mathbf{U}_{\mathrm{h}}:=\left[\mathbf{u}_{\mathrm{h}}, p_{\mathrm{h}}\right]^{t} \in \mathscr{W}_{\mathrm{h}, 0}$.

\section{Sub-grid scale method}

The basic idea of the sub-grid scale method is to approximate the effect of the component of the continuous solution which can not be resolved by the finite element mesh on the discrete finite element solution. This method was introduced by Hughes in [5] for the scalar convection-diffusion equation. This concept will be used here to stabilize the elastic incompressible problem in solid mechanics, allowing the use of linear/

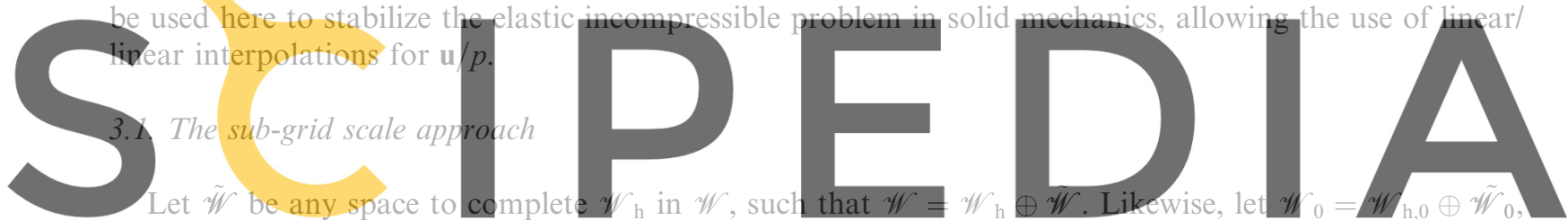

with $\tilde{\mathbb{W}}_{0}$ any space to complete $\mathscr{W}_{\mathrm{h} .0}$ in $\mathscr{W}_{0}$. The space $\tilde{\mathbb{W}}_{0}$ will be called the space of sub-grid scales or sub-

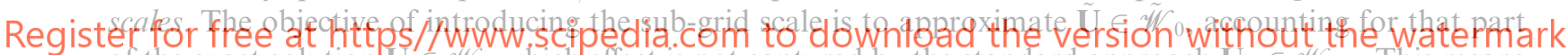

of the exact solution $\in \mathbb{W}_{0}$ which effect is not captured by the standard approach $\mathbb{U}_{\mathrm{h}} \in \mathscr{W}_{\mathrm{h}, 0}$. This means

that within this method the exact solution is now approximated by $\mathbb{U}=\mathbb{U}_{\mathrm{h}}+\tilde{\mathbf{U}}$. Thus, the continuous

problem (16) transforms into finding $\mathbf{U}_{\mathrm{h}} \in \mathscr{W}_{\mathrm{h}, 0}$ and $\mathbf{U} \in \tilde{W}_{0}$ such that:

$$
\begin{aligned}
& B\left(\mathbf{U}_{\mathrm{h}}, \mathbf{V}_{\mathrm{h}}\right)+B\left(\tilde{\mathbf{U}}, \mathbf{V}_{\mathrm{h}}\right)=L\left(\mathbf{V}_{\mathrm{h}}\right) \quad \forall \mathbf{V}_{\mathrm{h}} \in \mathscr{W}_{\mathrm{h}, 0}, \\
& B\left(\mathbf{U}_{\mathrm{h}}, \tilde{\mathbf{V}}\right)+B(\tilde{\mathbf{U}}, \tilde{\mathbf{V}})=L(\tilde{\mathbf{V}}) \quad \forall \tilde{\mathbf{V}} \in \tilde{\mathscr{W}}_{0} .
\end{aligned}
$$

Integrating by parts in (19) and (20) within each element, and introducing the notation $\int_{\Omega^{\prime}}:=\sum_{e=1}^{n_{\mathrm{elm}}} \int_{\Omega^{e}}$ and $\int_{\partial \Omega^{\prime}}:=\sum_{e=1}^{n_{\mathrm{elm}}} \int_{\partial \Omega^{e}}$, where $n_{\mathrm{elm}}$ is the number of elements of the finite element partition, these two equations can be written as:

$$
\begin{aligned}
& B\left(\mathbf{U}_{\mathrm{h}}, \mathbf{V}_{\mathrm{h}}\right)+\int_{\Omega^{\prime}} \tilde{\mathbf{U}} \cdot \mathscr{L}^{*}\left(\mathbf{V}_{\mathrm{h}}\right) \mathrm{d} \Omega+\int_{\partial \Omega^{\prime}} \tilde{\mathbf{u}} \cdot\left(\boldsymbol{\sigma}\left(\mathbf{V}_{\mathrm{h}}\right) \cdot n\right) \mathrm{d} \Gamma=L\left(\mathbf{V}_{\mathrm{h}}\right), \\
& \int_{\Omega^{\prime}} \tilde{\mathbf{V}} \cdot \mathscr{L}(\tilde{\mathbf{U}}) \mathrm{d} \Omega+\int_{\partial \Omega^{\prime}} \tilde{\mathbf{v}} \cdot\left(\boldsymbol{\sigma}\left(\mathbf{U}_{\mathrm{h}}+\tilde{\mathbf{U}}\right) \cdot \mathbf{n}\right) \mathrm{d} \Gamma=\int_{\Omega^{\prime}} \tilde{\mathbf{V}} \cdot\left[\mathbf{F}-\mathscr{L}\left(\mathbf{U}_{\mathrm{h}}\right)\right] \mathrm{d} \Omega,
\end{aligned}
$$

where $\mathbf{n}$ is the unit normal exterior to the integration domain, $(\boldsymbol{\sigma}(\cdot) \cdot \mathbf{n})$ stands for the tractions vector and $\mathscr{L}^{*}$ is the formal adjoint of the operator $\mathscr{L}$, given by:

$$
\mathscr{L}^{*}\left(\mathbf{V}_{\mathrm{h}}\right)=\left[\begin{array}{c}
-\nabla q_{\mathrm{h}}-2 \mu \nabla \cdot \operatorname{dev}\left[\nabla^{s} \mathbf{v}_{\mathrm{h}}\right] \\
-\frac{1}{K} q_{\mathrm{h}}+\nabla \cdot \mathbf{v}_{\mathrm{h}}
\end{array}\right] .
$$


Observe that $\boldsymbol{\sigma}\left(\mathbf{U}_{\mathrm{h}}+\tilde{\mathbf{U}}\right) \cdot \mathbf{n}$ represents the exact tractions, assumed to be continuous across interelement boundaries, and thus the sum across these boundaries is null. Thus, the second term in Eq. (22) vanishes, leading to the following equivalent expression for a specific $\mathbf{V}_{\mathrm{h}, \text { ort }} \in \tilde{\mathscr{W}}_{0}^{\perp}$

$$
\mathscr{L}(\tilde{\mathbf{U}})=\left[\mathbf{F}-\mathscr{L}\left(\mathbf{U}_{\mathrm{h}}\right)\right]+\mathbf{V}_{\mathrm{h}, \text { ort }} .
$$

This equation is the key point to capture the effects of the component $\tilde{\mathbf{U}}$ used to enrich the standard finite element solution $\mathbf{U}_{\mathrm{h}}$. Indeed, the objective is to account for this effects in the momentum Eq. (21) rather than to calculate $\tilde{\mathbf{U}}$. Thus, the following simplification could be considered

$$
\mathscr{L}(\tilde{\mathbf{U}}) \approx \tau^{-1} \tilde{\mathbf{U}}, \quad \tilde{\mathbf{U}} \in \tilde{\mathscr{W}}_{0}
$$

where $\tau$ is a $n_{\text {unk }} \times n_{\text {unk }}$ matrix defined within each element domain and referred to as the matrix of stabilization parameters which depends on the coefficients of $\mathscr{L}$.

We will consider only the integrals over element domains and neglect the effect of the integrals over element faces. This is as much as assume that $\tilde{\mathbf{U}}$ vanishes on the element boundaries. Thus, Eq. (21) can be written as

$$
B\left(\mathbf{U}_{\mathrm{h}}, \mathbf{V}_{\mathrm{h}}\right)+\int_{\Omega^{\prime}} \mathscr{L}^{*}\left(\mathbf{V}_{\mathrm{h}}\right) \cdot \tilde{\mathbf{U}} \mathrm{d} \Omega=L\left(\mathbf{V}_{\mathrm{h}}\right)
$$

which is the general expression of the stabilized method. Observe that there is an additional stabilizing term if compared to the standard formulation represented by (18).

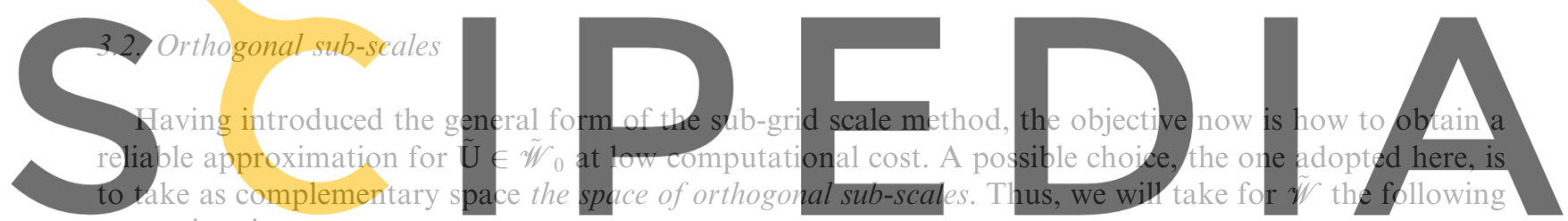

approximation:

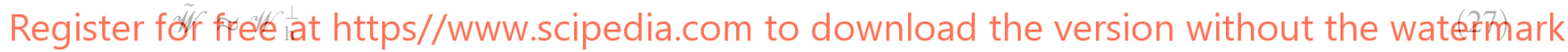

together with the assumption that $\tilde{\mathbb{W}}_{0} \approx \tilde{\mathbb{W}}$. This idea was first introduced by Codina in [2] as an extension of a stabilization method formulated for the Stokes problem in [4]. Therefore

$$
\tilde{\mathscr{W}}_{0} \approx \tilde{\mathscr{W}} \approx \mathscr{W}_{\mathrm{h}}^{\perp}
$$

Thus, if we call $P_{\mathrm{h}}$ the $L_{2}$ orthogonal projection onto $\mathscr{W}_{\mathrm{h}}$, and $P_{\mathrm{h}}^{\perp}$ the orthogonal projection onto $\mathscr{W}_{\mathrm{h}}^{\perp}$, after imposing that $\tilde{\mathbf{U}} \in \tilde{\mathscr{W}}_{\mathrm{h}}^{\perp}$, one can find the following approximations

$$
\tilde{\mathbf{U}}=P_{\mathrm{h}}^{\perp}\left\{\tau\left[\mathbf{F}-\mathscr{L}\left(\mathbf{U}_{\mathrm{h}}\right)\right]\right\} \in \mathscr{W}_{\mathrm{h}}^{\perp} .
$$

As we will show, a definition of $\tau$, the matrix of stabilization parameters as

$$
\tau=\operatorname{diag}(\underbrace{\tau, \ldots, \tau}_{n_{\text {dim }}}, 0)
$$

leads to satisfactory results in the case of the incompressible elasticity problem. The value of $\tau$ has been studied by many authors for the solution of fluid-mechanics problems, either by convergence analysis or, more recently, by means of Fourier analysis [3]. In a solid-mechanics context, following similar guidelines, one can find that the corresponding value of $\tau$ can be taken as:

$$
\tau=c\left(\frac{2 \mu}{h^{2}}\right)^{-1},
$$


where $c$ is a numerical constant and $h$ is a characteristic length of the element. Observe that to consider the pressure component in (30) equal to zero is equivalent to neglect the sub-scale component of the pressure field.

It must be pointed out that $\mathbf{F}$ in (29) belongs to the space $\mathscr{W}_{\mathrm{h}}$, therefore $P_{\mathrm{h}}^{\perp}(\mathbf{F})=\mathbf{0}$. On the other hand, $\nabla \cdot \nabla \mathbf{u}_{\mathrm{h}}$ and $\nabla \cdot \nabla \mathbf{v}_{\mathrm{h}}$ in (14) and (23), involve second derivatives of finite element functions which vanish when linear elements are used. In case of higher order elements these derivatives can be neglected in computing $\tilde{\mathbf{U}}$, leading to a method which is still consistent [3].

If we assume that the orthogonal projection of a variable $(\cdot)$ can be computed as: $P_{\mathrm{h}}^{\perp}(\cdot)=(\cdot)-P_{\mathrm{h}}(\cdot)$, the components of vector $\tilde{\mathbf{U}}$ transforms in

$$
\begin{aligned}
& \tilde{\mathbf{u}}=\tau_{e}\left(\nabla p_{\mathrm{h}}-P_{\mathrm{h}}\left(\nabla p_{\mathrm{h}}\right)\right), \\
& \tilde{p}=0 .
\end{aligned}
$$

Inserting this result in (26), a computable expression for the stabilization term can be obtained as

$$
\int_{\Omega^{\prime}} \mathscr{L}^{*}\left(\mathbf{V}_{\mathrm{h}}\right) \cdot \tilde{\mathbf{U}} \mathrm{d} \Omega=\int_{\Omega^{\prime}} \tau\left(-\nabla q_{\mathrm{h}} \cdot P_{\mathrm{h}}^{\perp}\left(\nabla p_{\mathrm{h}}\right)\right) \mathrm{d} \Omega=\int_{\Omega^{\prime}} \tau\left(-\nabla q_{\mathrm{h}} \cdot\left[\nabla p_{\mathrm{h}}-P_{\mathrm{h}}\left(\nabla p_{\mathrm{h}}\right)\right]\right) \mathrm{d} \Omega .
$$

Calling $\Pi_{\mathrm{h}}=P_{\mathrm{h}}\left(\nabla p_{\mathrm{h}}\right)$, we can rewrite the last expression as:

$\int_{\Omega^{\prime}} \mathscr{L}^{*}\left(\mathbf{V}_{\mathrm{h}}\right) \cdot \tilde{\mathbf{U}} \mathrm{d} \Omega=\sum_{e=1}^{n_{\mathrm{elm}}} \tau_{e} \int_{\Omega^{e}}-\nabla q_{\mathrm{h}} \cdot\left[\nabla p_{\mathrm{h}}-\Pi_{\mathrm{h}}\right] \mathrm{d} \Omega$,

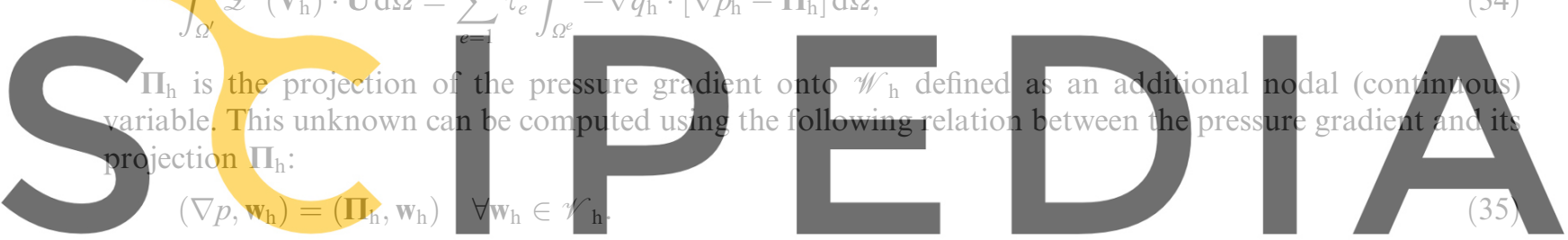

Finally, we can write the stabilized version of problem (8) and (9), together with the boundary conditions

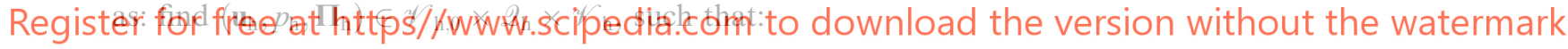

$$
\begin{aligned}
& a\left(\mathbf{u}_{\mathrm{h}}, \mathbf{v}_{\mathrm{h}}\right)+\left(p_{\mathrm{h}}, \nabla \cdot \mathbf{v}_{\mathrm{h}}\right)=L\left(\mathbf{v}_{\mathrm{h}}\right) \quad \forall \mathbf{v}_{\mathrm{h}} \in \mathscr{V}_{\mathrm{h}, 0}, \\
& \left(\nabla \cdot \mathbf{u}_{\mathrm{h}}, q_{\mathrm{h}}\right)-\left(\frac{1}{K} p_{\mathrm{h}}, q_{\mathrm{h}}\right)-\sum_{e=1}^{n_{\mathrm{elm}}} \tau_{e} \int_{\Omega^{e}} \nabla q_{\mathrm{h}} \cdot\left[\nabla p_{\mathrm{h}}-\boldsymbol{\Pi}_{\mathrm{h}}\right] \mathrm{d} \Omega=0 \quad \forall q_{\mathrm{h}} \in \mathscr{Q}_{\mathrm{h}}, \\
& \left(\nabla p_{\mathrm{h}}, \mathbf{w}_{\mathrm{h}}\right)-\left(\boldsymbol{\Pi}_{\mathrm{h}}, \mathbf{w}_{\mathrm{h}}\right)=0 \quad \forall \mathbf{w}_{\mathrm{h}} \in \mathscr{V}_{\mathrm{h}} .
\end{aligned}
$$

\section{Implementation aspects}

To obtain the matrix structure of the stabilized system of equations proposed in (36), let us firstly define the elemental matrix $\mathbf{K}^{(e)}=\left[\mathbf{K}^{\mathrm{AB}}\right]^{(e)}$

$$
\left[\mathbf{K}^{\mathrm{AB}}\right]^{(e)}=\left[\begin{array}{ccc}
\mathbf{K}_{\mathrm{dev}}^{\mathrm{AB}} & \mathbf{G}^{\mathrm{AB}} & \mathbf{0} \\
\left(\mathbf{G}^{\mathrm{AB}}\right)^{\mathrm{T}} & \left(-\frac{1}{K} M_{\mathrm{p}}^{\mathrm{AB}}-\tau_{e} L^{\mathrm{AB}}\right) & \tau_{e}\left(\mathbf{G}^{\mathrm{AB}}\right)^{\mathrm{T}} \\
\mathbf{0} & \tau_{e} \mathbf{G}^{\mathrm{AB}} & -\tau_{e} \mathbf{M}^{\mathrm{AB}}
\end{array}\right],
$$

where the entry $(\cdot)^{\mathrm{AB}}$ is a sub-matrix corresponding to the local nodes $\mathrm{A}$ and $\mathrm{B}$. In Eq. (37), $\mathbf{K}_{\mathrm{dev}}^{\mathrm{AB}}$ is the deviatoric component of the standard elastic stiffness matrix defined as:

$$
\mathbf{K}_{\mathrm{dev}}^{\mathrm{AB}}=\int_{\Omega_{e}} \mathbf{B}_{\mathrm{A}}^{\mathrm{T}} \mathbf{D}_{\mathrm{dev}} \mathbf{B}_{\mathrm{B}} \mathrm{d} \Omega,
$$


where $\mathbf{D}_{\mathrm{dev}}$ is the deviatoric constitutive matrix and $\mathbf{B}$ is a standard deformation sub-matrix. The generic term of the discrete gradient matrix operator $\mathbf{G}^{\mathrm{AB}}$ is given by:

$$
\mathbf{G}^{\mathrm{AB}}=\int_{\Omega_{e}}\left[\nabla N^{\mathrm{A}}\right] N^{\mathrm{B}} \mathrm{d} \Omega, \quad \text { where } \quad\left[\nabla N^{\mathrm{A}}\right]=\left[N_{, x}^{\mathrm{A}} N_{, y}^{\mathrm{A}} N_{, z}^{\mathrm{A}}\right]^{\mathrm{T}}
$$

while the laplacian term $L^{\mathrm{AB}}$ can be expressed as:

$$
L^{\mathrm{AB}}=\int_{\Omega_{e}}\left[\nabla N^{\mathrm{A}}\right]^{\mathrm{T}}\left[\nabla N^{\mathrm{B}}\right] \mathrm{d} \Omega .
$$

Finally $M_{\mathrm{p}}^{\mathrm{AB}}$ and $\mathbf{M}^{\mathrm{AB}}$ are the "mass" matrices associated to the pressure and displacement fields, respectively:

$$
\begin{aligned}
& M_{\mathrm{p}}^{\mathrm{AB}}=\int_{\Omega_{e}} N^{\mathrm{A}} N^{\mathrm{B}} \mathrm{d} \Omega, \\
& \mathbf{M}^{\mathrm{AB}}=\left(\int_{\Omega_{e}} N^{\mathrm{A}} N^{\mathrm{B}} \mathrm{d} \Omega\right) \delta_{i j} \quad i, j=1, \ldots, 3 .
\end{aligned}
$$

The associated global matrix structure of problem (36) is:

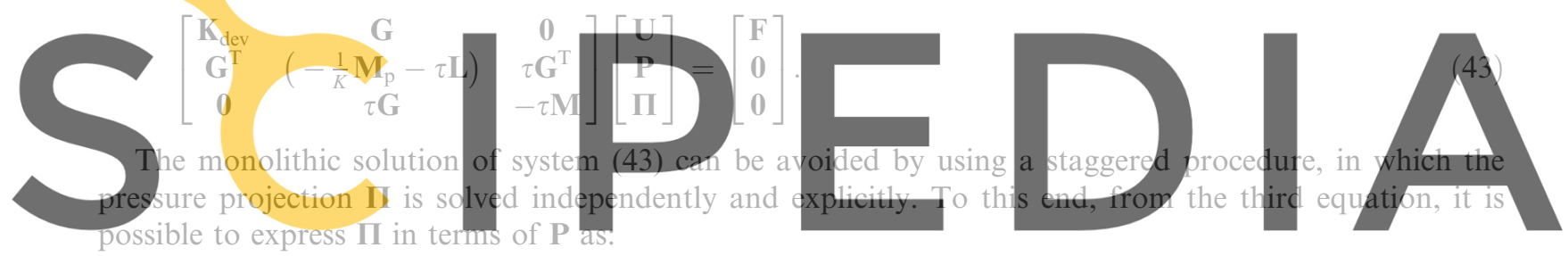

\section{Register for free af https//www.scipedia.com to download the version without the watef'mark}

Substituting $\mathbb{I}$ into the second equation it is possible to formally condensate $\mathbb{\Pi}$, to obtain a solving system only in terms of the nodal displacements and pressures, as:

$$
\left[\begin{array}{cc}
\mathbf{K}_{\mathrm{dev}} & \mathbf{G} \\
\mathbf{G}^{\mathrm{T}} & -\frac{1}{K} \mathbf{M}_{\mathrm{p}}-\tau\left(\mathbf{L}-\mathbf{G}^{\mathrm{T}} \mathbf{M}^{-1} \mathbf{G}\right)
\end{array}\right]\left[\begin{array}{l}
\mathbf{U} \\
\mathbf{P}
\end{array}\right]=\left[\begin{array}{l}
\mathbf{F} \\
\mathbf{0}
\end{array}\right]
$$

Observe that the stabilization effect of the proposed technique, formally reduces to a stability term given by $\tau\left(\mathbf{L}-\mathbf{G}^{\mathrm{T}} \mathbf{M}^{-1} \mathbf{G}\right)$.

From the computational point of view, this suggests an iterative solution of the problem. In a first step the nodal displacements and pressure fields $\mathbf{U}^{(i)}$ and $\mathbf{P}^{(i)}$ respectively, are computed using the previous iteration value of the projected pressure gradient $\Pi^{(i-1)}$ in the RHS. In the next step it is possible to compute the projections $\boldsymbol{\Pi}^{(i)}$ in terms of current values of the pressure field. The resulting iterative algorithm is the following:

Box 1: Algorithm to solve the stabilized system.

Solve at global level $\mathbf{U}^{(i)}$ and $\mathbf{P}^{(i)}:\left[\begin{array}{cc}\mathbf{K}_{\mathrm{dev}} & \mathbf{G} \\ \mathbf{G}^{\mathrm{T}} & -\frac{1}{K} \mathbf{M}_{\mathrm{p}}-\tau \mathbf{L}\end{array}\right]\left[\begin{array}{l}\mathbf{U}^{(i)} \\ \mathbf{P}^{(i)}\end{array}\right]=\left[\begin{array}{c}\mathbf{F} \\ -\tau \mathbf{G}^{\mathrm{T}} \cdot \mathbf{\Pi}^{(i-1)}\end{array}\right]$

Compute and store: $\boldsymbol{\Pi}^{(i)}=\mathbf{M}^{-1}\left(\mathbf{G P}^{(i)}\right)$

Perform next iteration: $i \leftarrow i+1$ 
Note that the computation of the projections $\Pi$ can be transformed in a trivial system by considering the lumped mass matrix as an approximation to M. Note also that the computational cost due to the iterative algorithm proposed is negligible in a nonlinear context. In fact, in this case the equilibrium iterations induced by the nonlinearity are also used to compute the projected pressure gradient.

\section{Numerical results}

In this section the formulation proposed is validated assuming both compressibility and incompressibility conditions. The stabilized system of Eq. (36) is solved using the algorithm presented in Box-1. Linear elastic constitutive behaviour is assumed. Performance of the method is tested considering both a 2D plane-strain triangular mesh and a 3D tetrahedral mesh.

\subsection{Plane strain Cook's membrane problem}

The Cook's membrane problem is a bending dominated example that has been used by many authors as a reference test to check their element formulations, see: [7,9] among others. Here it will be used to compare results for compressible and incompressible elasticity, showing the behaviour of both quadrilateral and triangular elements. As reference solution the mixed mean dilatation/pressure element, generally referred as Q1P0 (see [10]), is used. The problem consists in a tapered panel, clamped on one side and subjected to a

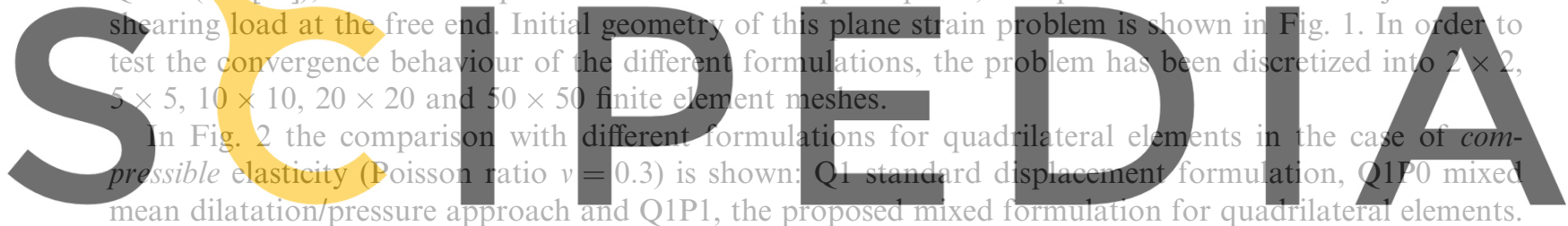

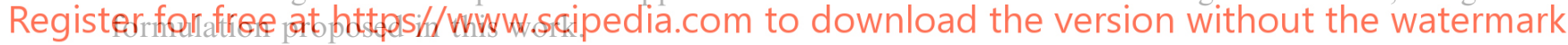

Fig. 3 shows the behaviour of both quadrilateral and triangular elements in the case of near incompressible elasticity (Poisson ratio $\nu=0.4999$ ). Observe how the proposed formulation converges faster to the exact solution than Q1P0 mixed approach, even if triangular meshes are used. The figure also shows the poor performance of the Q1 and T1 standard elements within the context of nearly incompressible elasticity, due to an extreme locking effect.
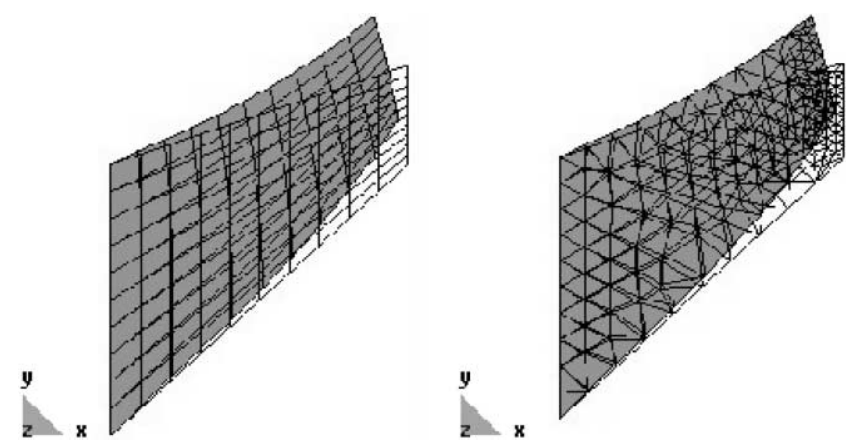

Fig. 1. Plane strain Cook's membrane problem: original and deformed geometries for triangular and quadrilateral meshes. 
PLANE STRAIN COOK'S MEMBRANE PROBLEM: Compressible case (Poisson's ratio $=0.3$ ) Top Corner Displacement [mm]

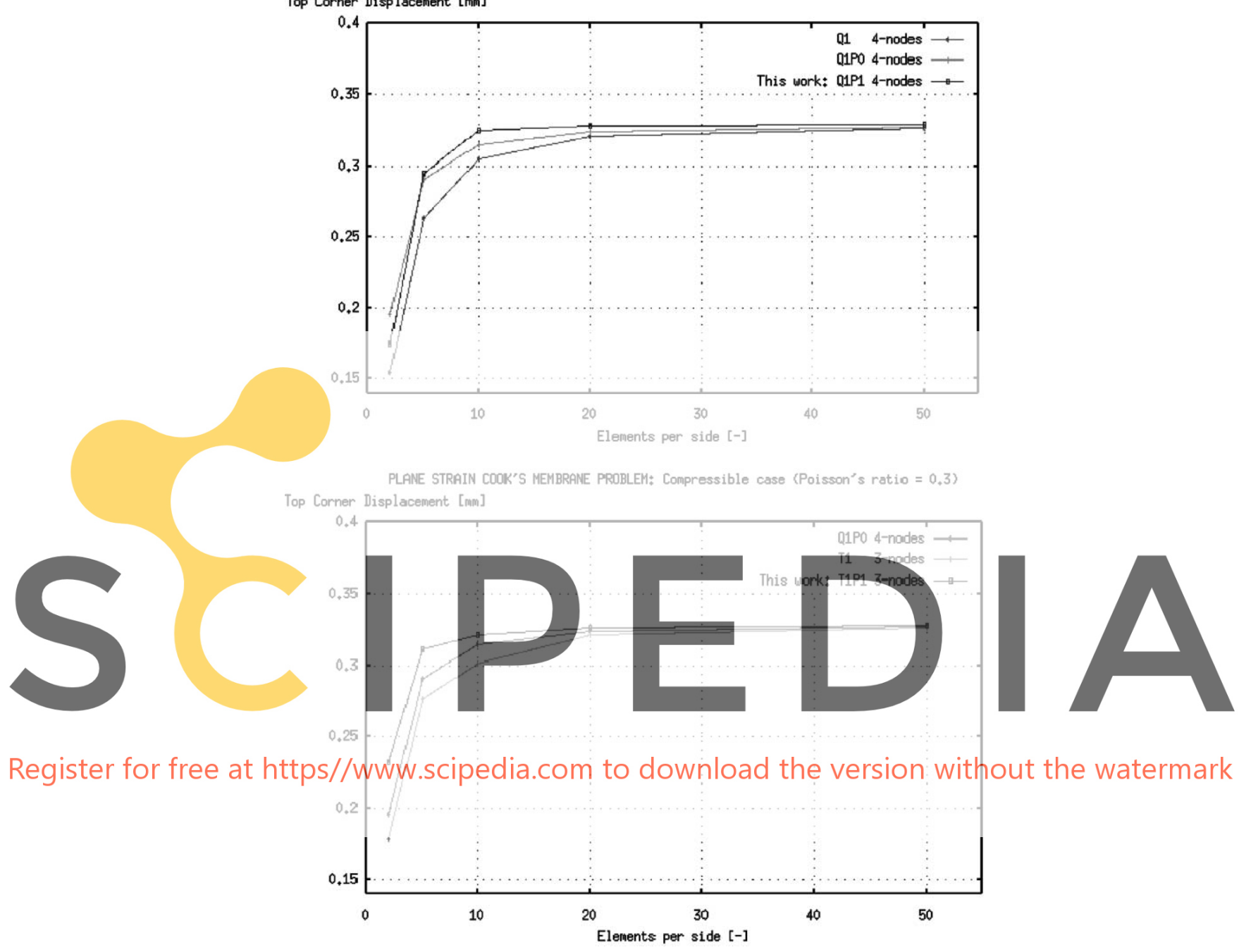

Fig. 2. Plane strain Cook's membrane problem: convergence of different element formulations for compressible elasticity (Poisson ratio $=0.3)$. $(\mathrm{Q} 1)$ : standard displacement model for quadrilateral elements, $(\mathrm{T} 1)$ : standard displacement model for triangular elements, (Q1P0): mixed mean dilatation/pressure approach for quadrilateral elements, (Q1P1): proposed mixed formulation for quadrilateral elements and (T1P1): proposed mixed formulation for triangular elements.

\subsection{Upsetting problem}

The purpose of this example is to illustrate that, within the formulation presented, a suitable treatment of near incompressible elasticity is obtained even if coarse tetrahedral meshes are used. The example consists of an upsetting problem where a $14 \times 14 \times 10$ specimen is deformed up to $7 \%$ of its height. The upper head is assumed to be perfectly fixed to a rigid plate which moves downward producing increasing compression. The bottom surface displacements are prescribed to zero. A small strain linear elastic 


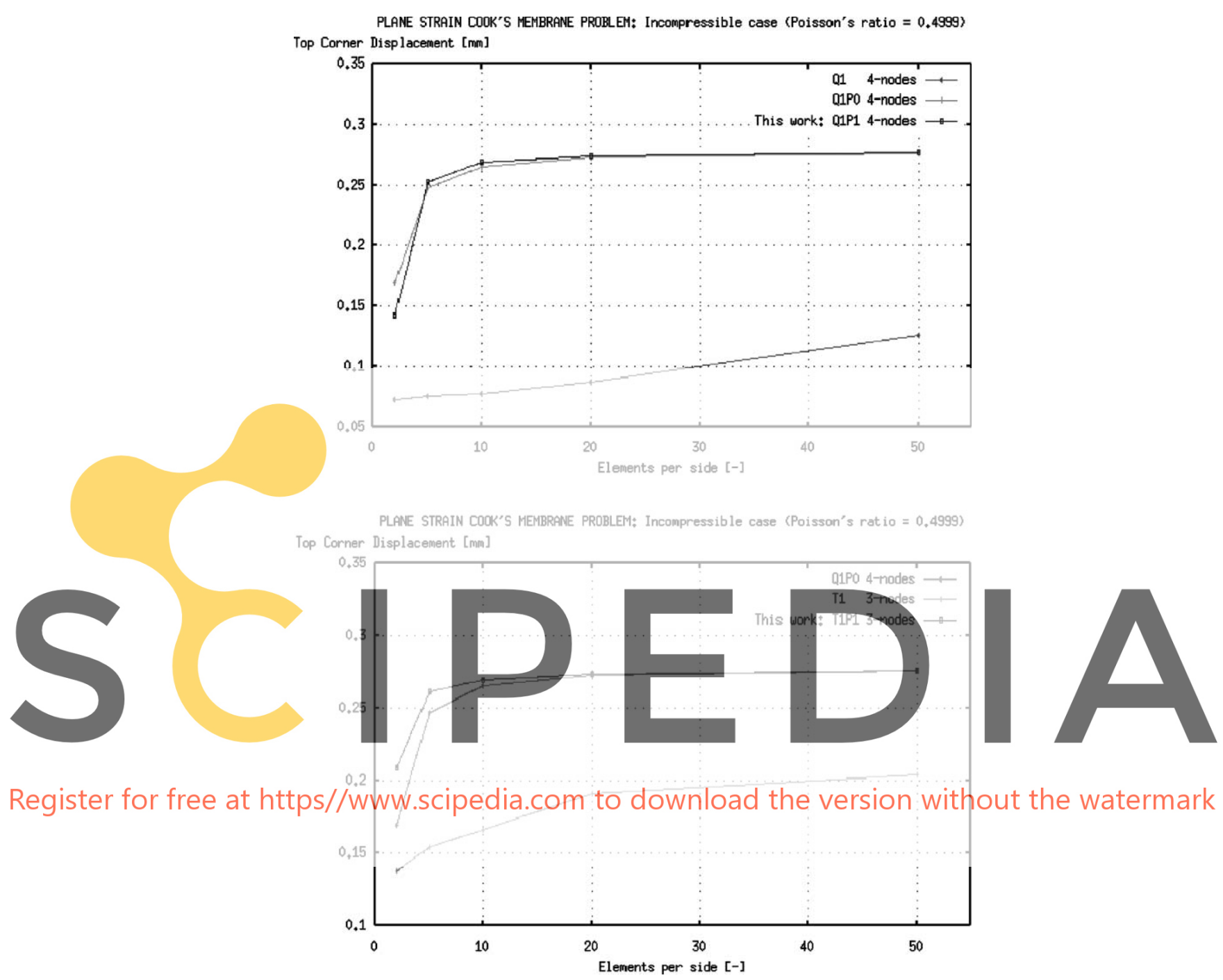

Fig. 3. Plane strain Cook's membrane problem: convergence of different element formulations for near incompressible elasticity (Poisson ratio $=0.4999)$. $(\mathrm{Q} 1)$ : standard displacement model for quadrilateral elements, $(\mathrm{T} 1)$ : standard displacement model for triangular elements, (Q1P0): mixed mean dilatation/pressure approach for quadrilateral elements, (Q1P1): proposed mixed formulation for quadrilateral elements and (T1P1): proposed mixed formulation for triangular elements.

constitutive model is used: Young's modulus is set to $2.0 E+5 \mathrm{Mpa}$ and to achieve the volume preserving constraint Poisson's ratio is fixed to 0.4999 .

Fig. 4 shows the behaviour obtained using the different formulations. We may observe the similarity, in terms of deformed shapes, between the Q1P0 formulation and the proposed stabilized formulation. On the other hand, the locking behaviour of the standard formulation for tetrahedral elements is also evident. The stress response using the different formulations is presented in terms of both $\mathrm{J} 2$-von Mises deviatoric stress indicator and pressure variable contour fills. Also in this case the similarity between the Q1P0 formulation and the proposed stabilized formulation can be observed and compared to the response of a standard formulation for tetrahedra elements, which exhibits a severe locking effect. 


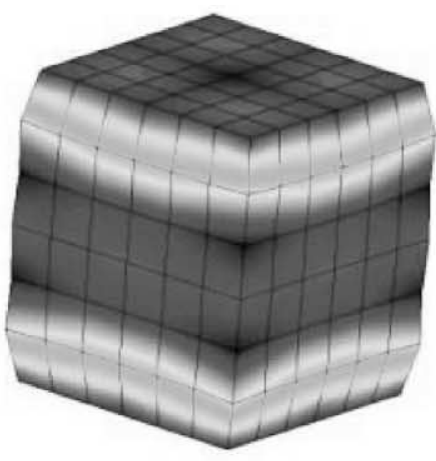

\section{Q1P0 Formulation}
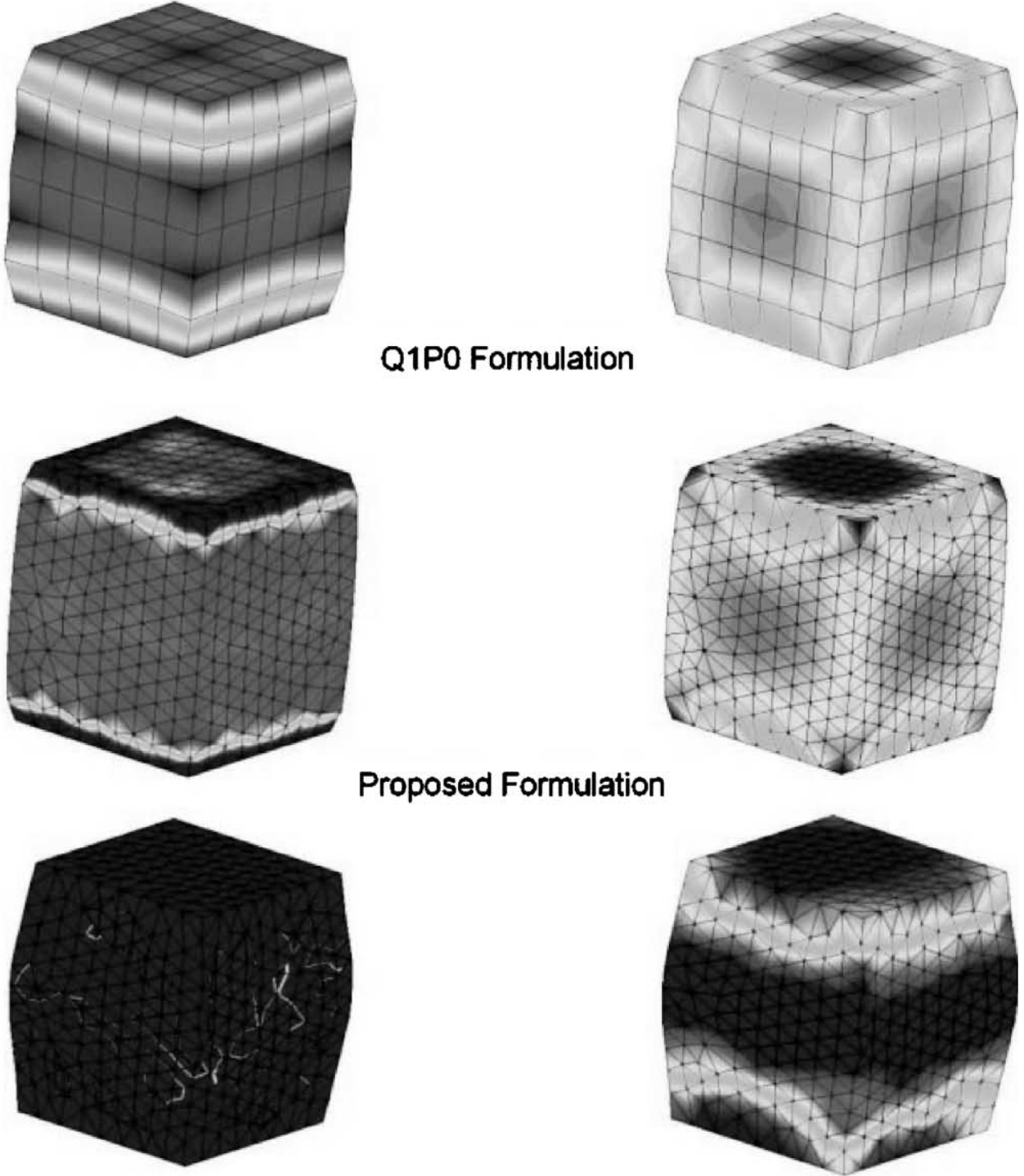

\section{Proposed Formulation}

\section{Standard Formulation}
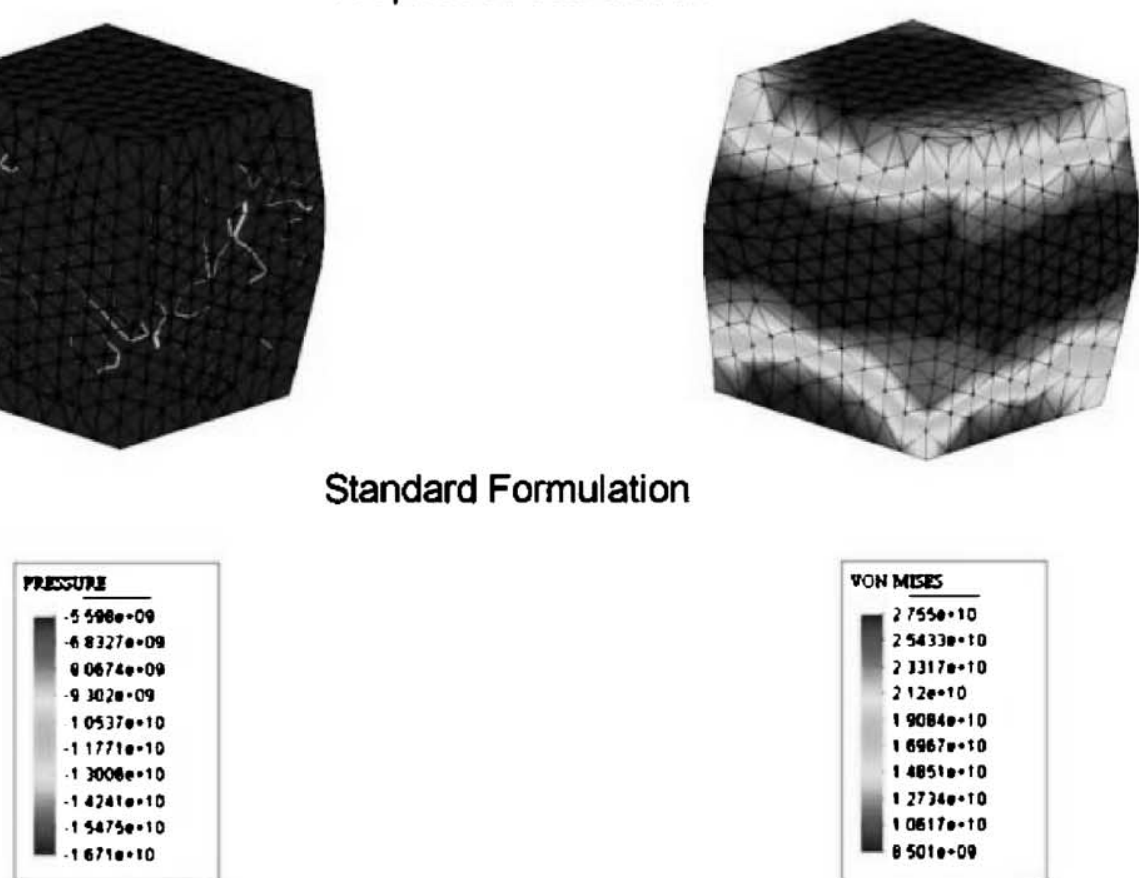

Fig. 4. Elastic upsetting problem: pressure and J2 von Mises deviatoric stress indicator contour fills achieved with different formulations. (a) Hexahedral mesh with a Q1P0 formulation, (b) tetrahedral mesh using the proposed stabilized formulation and (c) tetrahedral mesh with standard formulation. 


\section{Conclusions}

In this paper a stabilized finite element method to deal with incompressibility in solid mechanics problems is presented. The method is based on the concept of orthogonal sub-scales decomposition and overcomes the Babuška-Brezzi condition allowing an accurate and stable mixed formulation with equal order $\mathbf{u} / p$ interpolations. The method presented is suitable not only for quadrilateral and hexahedral elements but, interestingly, for triangular and tetrahedral elements as well. The drawback is the computational cost, due to the iterative algorithm introduced to avoid the monolithic solution. The formulation, which has been presented here for incompressible elasticity, appears to be appropriate for elasto-plastic analysis of metals by means of the $\mathbf{J} 2$-model as well, due to the decoupled characteristic of this constitutive model. The ultimate goal is to extend this method to the numerical simulation of rubber materials or bulk metal forming processes. Generalization of this work in the context of elasto-plasticity will be published soon.

\section{Acknowledgements}

The authors are thankful to Professor Ramon Codina and Dr. Alfredo Huespe for many useful discussions.

\section{References}

[1] F. Brezzi, M. Fortin, Mixed and hybrid finite element methods, Spinger, New York, 1991.

[2] R. Codina, Stabilization of incompressibility and convection through orthogonal sub-scales in finite element methods, Comp. Meth. in Appl. Mech. Engrg. 190 (2000) 1579-1599.

[3] R. Codina, Stabilized finite element approximation of transient incompressible flows using orthogonal sub-scales, Comp. Meth. in Appl. Mech. and Engrg. 191 (2002) 4295-4321.

[4] R. Codina, J. Blasco, Stabilized finite element method for transient Navier-Stokes equations based on pressure gradient projection, Comp. Meth. in Appl. Mech. Engrg. 182 (2000) 287-300.

[5] T.J.R. Hughes, Multiscale phenomena: Green's function Dirichlet-to Neumann formulation, subgrid scale models, bubbles and the origins of stabilized formulations, Comp. Meth. Appl. Mech. Engrg. 127 (1995) 387-401.

[6] O. Klaas, A. Maniatty, M.S. Shephard, A stabilized mixed finite element method for finite elasticity. Formulation for linear displacement and pressure interpolation, Comp. Meth. Appl. Mech. Engrg. 180 (1999) 65-79.

[7] C. Miehe, Aspects of the formulation and finite element implementation of large strain isotropic elasticity, Int. J. Num. Meth. Engrg. 37 (1994) 1981-2004.

[8] E. Oñate, J. Rojek, R.L. Taylor, O.C. Zienkiewicz, Linear triangles and tetrahedra for incompressible problem using a finite calculus formulation, Proceeding of European Conference on Computational Mechanics, ECCM, 2001.

[9] J.C. Simo, M.S. Rifai, A class of mixed assumed strain methods and the method of incompatible modes, Int. J. Num. Meth. Engrg. 29 (1990) 1595-1638.

[10] J.C. Simo, R.L. Taylor, K.S. Pister, Variational and projection methods for the volume constraint in finite deformation elastoplasticity, Comp. Meth. Appl. Mech. Engrg. 51 (1985) 177-208.

[11] R.L. Taylor, Mixed formulation for triangular and tetrahedral finite elements, Conference proceeding, Metodos Numericos en la Ingenieria, SEMNI, 1999.

[12] O.C. Zienkiewicz, J. Rojek, R.L. Taylor, M. Pastor, Triangles and tetrahedra in explicit dynamic codes for solids, Int. J. Num. Meth. Engrg. 43 (1998) 565-583. 